\title{
Design and Implement of App Based OBD-II Vehicle Tracking System
}

\author{
Yen-Jen Chen ${ }^{+}$and Ching-Chuan Liu \\ Department of Electronics Engineering, Ming Chi University of Technology
}

\begin{abstract}
The fleet management service identifies the driver behavior pattern, restricts improper operation and avoids trouble-making actions or deviant behavior to save operation costs. The fleet management system architecture mostly depends on hardware driving recorders to obtain relevant data. However, the hardware system is not easy to customize, expand or adjust due to its fixed data categories and system functions. Therefore, by integrating OBD-II and smart phones, this paper proposes developing a software vehicle tracking system with an algorithm to detect vehicle-environment interactive behaviors based on Android, an open source software stack. This system aims to collect various information from vehicles and mobile phone sensors, identify interactive behavior, provide the back-end vehicle management system for monitoring and analysis, improve traditional fleet management in terms of customization, expansion and real-time performance, and facilitate fast-speed establishment and customization of the driving management system.
\end{abstract}

Keywords: fleet management, vehicle tracking system, OBD-II, Apps, vehicle behaviour analysis.

\section{Introduction}

Fig. 1 shows the operation architecture of the vehicle tracking system (VTS) currently used to grasp vehicle information. It generally integrates the OBD-II [1] reader, GPS (Global Positioning System) and wireless communication chip. The vehicle tracking system reads the OBD-II data and GPS latitude and longitude coordinates in the on-board computer on a regular basis. It then sends the data regularly back to the back-end fleet management system (FMS) through GPRS (General Packet Radio Service), 3G (3rdGeneration), 4G (4th-Generation) technology, etc., for storage, analysis and processing. At present, many related on-board applications are being upgraded based on this architecture [2].

The trend of Internet of Things has further boosted the development of intelligent telematics technology. To assist with driving and avoid danger on one hand, and customize the business logic in support of fleet operation on the other hand. This study aims to support related on-board device system integrators for the integration of OBD-II and smartphone sensor interface. Specifically, in mobile phones a behavior detection and analysis software will be developed to provide real-time information about vehicle state and vehicleenvironment interactive behaviour. A set of software vehicle tracking system will be established for quick intelligent and customized performances and for more effective commercial fleet management.

\section{Related Work}

In recent years, the fleet management or related application system analyses the application state of vehicles in different fields through the information provided by OBD-II. Nevertheless, some major limitations have been gradually found in the traditional vehicle VTS due to its operation architecture (Figure 1). It's weak in OBD-II protocol support. It can acquire ten-odd types of diagnostic data about vehicles only. It lacks the function of expansion module. And it is unable to show the vehicle state in the front end in a timely manner. To improve such deficiencies, this study comes up with a vehicle system that will integrate the OBD-II, the inbuilt sensor of mobile phones, the transmission interface and the display screen.

\footnotetext{
+ Corresponding author. Tel.: +886-2-29089899.

E-mail address: yjchen@mail.mcut.edu.tw.
} 
Many researches have used ELM32X OBD-II diagnosis for related testing, since C.E. Lin et al. applied the GPRS surveillance technology for real-time remote fault diagnosis [3]. G. Albertengo et al., in cooperation with Telecom Italia, integrated the Bluetooth diagnostic device and GPS by using ELM327 to detect the arrival and departure of vehicles [4]. Among related researches on specific audio recognition, F. Beritelli et al. adopted the Fast Fourier Transform and Mel-Frequency Cepstral Coefficients to help the hearing impaired recognize emergency warning signals sent by tow trucks, police cars, ambulances and fire trucks [5]. Regarding the application of on-board sensors, J. Dai et al. detected the abnormal turns caused by drunk driving with the accelerometer and according to the ratios reflected by specific driving data [6].

All the above-mentioned researches used the data for real-time surveillance and management, but they focused on the analysis of information without combining others and failed to use all aspects of data. Targeted at these problems, this study combines OBD-II, sound-related information and mobile phone sensor data, and acquires a wider variety of information to judge vehicle-environment interactive behavior.

\section{System Architecture}

This study presents an overall architecture of a software VTS, as shown in Fig. 2. The smart phone will connect to OBD-II via Bluetooth and automatically issue a command for the diagnostic device to capture the on-board computer data. After acquiring the data, the OBD-II sends the diagnostic results back to the smart phone for subsequent processing. On the other hand, the inbuilt sensors can provide extra data of G-Sensor, Magnetic-Sensor etc. The vehicle and sensor data coupled with the processing power of the smart phone can actualize the real-time on-board analysis, processing, state recognition, storage, display and transmission.

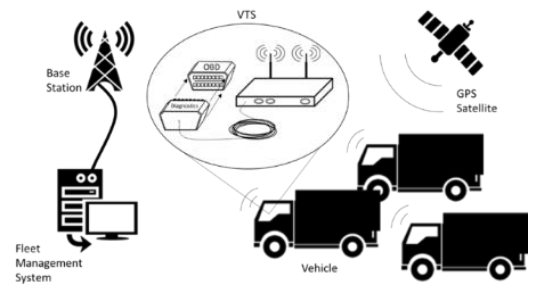

Fig. 1: Operation Architecture of Traditional VTS.

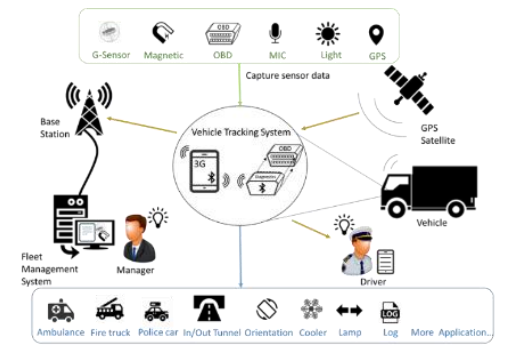

Fig. 2: The Proposed VTS Architecture.

\section{Application Data Processing}

\subsection{OBD-II Data processing}

The OBD-II information obtained by this study through ELM327 includes the OBD-II interface voltage, real-time diagnostic data of the power system, and error codes, etc. Besides, during the vehicle tracking system initialization, the ELM327 diagnostic device is set via the AT command to add OBD-II headers into the transmitted OBD-II data formats, so that this vehicle tracking system can identifying the protocol type, distinguishing the sources of OBD-II data and handle the data reorganization.

\subsection{Mobile phone sensor data processing}

The mobile phone sensors include GPS, G-Sensor, magnetic sensor etc. Regarding the data of G-Sensor, magnetic sensor and MIC, the amount of data per second is relatively large. Moreover, these data mostly have to be processed first in order to become useful. Consequently, the original data of this sort of messages will not be stored. The ways to process these data are interpreted as follows.

- G-Sensor and three-axis magnetic sensor: The data is transformed into the direction angle, angles of depression and elevation, and roll angle by Android Application Programming Interface (API).

- G-Sensor and pitch angle: According to the extent of vehicle inclination, as show in Figure 3. The non-orthogonality of Y-coordinate and centripetal acceleration make it impossible to accurately indicate the vehicle's acceleration. This study then uses the pitch angles to correct the Y-coordinate acceleration value, as shown by Formula (1). Where, Gyo represents the corrected acceleration, $\mathrm{Gy}_{\mathrm{i}}$ represents the Y-coordinate acceleration and Oy represents the pitch angle. 
- MIC: The voice is obtained through MIC at a sampling frequency of $8,000 \mathrm{~Hz}$. A Fast Fourier transform is implemented using the method of Decimation-in-time (DIT) Radix-2 FFT [7] which is actualized through open source codes. Each second is segmented into sixteen voice slots (as shown in Figure 4) and respectively converted through FFT. The frequency characteristics in the sixteen voice slots are compared to see whether they conform to the target voice.

$$
\mathrm{Gy}_{\mathrm{o}}=\mathrm{Gy}_{\mathrm{i}}+(9.81 \times \sin (\mathrm{Oy})) \text {. }
$$

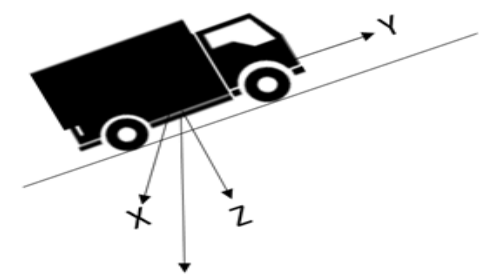

Fig. 3: The Corresponding Relationship between Smart Phone's Placement in the Vehicle and G-Sensor Data.

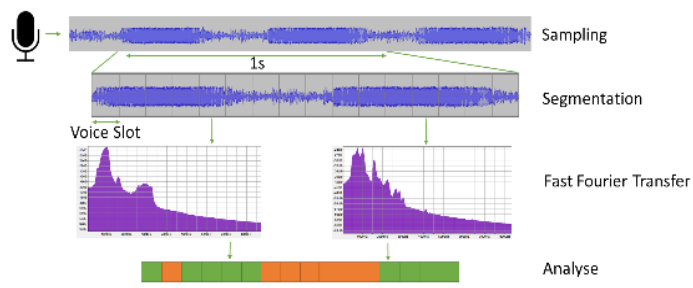

Fig. 4: A Diagram of Voice Analysis Procedure.

\section{State Identification}

While a VTS is processing the state information, the OBD-II and smart phone sensor data are received and then analyzed and processed. The algorithms to identify these state messages are illustrated as follows.

\subsection{The state of OBD-II messages}

Regarding OBD-II diagnostic messages, this paper takes the battery voltage and fuel level as two examples for illustration. The vehicle battery voltage varies according to the vehicle state. When the engine is shut down or turned on, the normal voltage will range from distinct voltage levels. The VTS detects the voltage value range in line with this feature and a warning message is provided in times of abnormal voltage.

The amount of fuel is judged based on the speed and fuel level provided by OBD-II. The VTS, through a much shorter sampling period, analyzes the fuel level at the zero speed or a stable speed to improve dramatic fuel level changes by vehicle sway. As the data of fuel amount is updated, the fuel level falling below set value will indicate a state of overly low fuel amount. If the fuel level is higher than set value of the recorded value, it will be regarded as refueling. At last, an abnormal decrease in the fuel level will indicate abnormal fuel amount. The estimated fuel consumption shown by Formula (2) is the benchmark for this judgement of abnormality. In the following formula, $\mathrm{EF}_{\mathrm{n}}$ represents the estimated fuel consumption, $\mathrm{EF}_{\mathrm{n}-1}$ represents the previous estimated fuel consumption and $\mathrm{RF}_{\mathrm{n}}$ represents the actual measured fuel consumption.

$$
\mathrm{EF}_{\mathrm{n}}=\mathrm{EF}_{\mathrm{n}-1} * \gamma+\mathrm{RF}_{\mathrm{n}} *(1-\gamma), 0<\gamma<1
$$

\subsection{Voice message state}

The relay for flash light in a vehicle sends out a voice lasting for a small period of time. The VTS captures two frequency bands that have distinct peak values. If both frequency bands manifest a distinct peak value simultaneously, it can be judged as the feature of flash voice. At last, the activation of flash voice is identified by calculating the consecutive number of times for the periodical appearance of this feature.

\subsection{Recognizing related states of mobile phone sensor messages}

Due to space limitation, this paper just expounds on vehicle left/right turn as an example. Two methods are offered to identify the message of left/right turn according to the value of the direction sensor. The first is the degree values in each set conform to the rule that a new set of data is larger/smaller than the old set with consecutive times. The second is identified by comparing the changes to new and old stable degrees.

\section{Result and Analysis}

This vehicle tracking system experiments on the Luxgen SUV as its test platform. As shown by the OBD-II data in Table 1, this vehicle acquires the messages provided by two electronic control units, and gets 
33 types of PIDs, 42 sets of OBD-II data in total. From the above-mentioned test result, it can be confirmed that this vehicle tracking system is capable of effectively processing the data sent by multiple ECUs, and automatically identifying the readable OBD-II data for further processing.

Table 1: A List of Diagnostics PIDs Successfully Acquired by Luxgen Five-seat Recreational Vehicle

\begin{tabular}{|c|c|c|c|c|c|c|c|c|c|c|c|c|c|c|c|c|c|}
\hline $\mathrm{ECU}$ & \multicolumn{17}{|c|}{ PID } \\
\hline \multirow{2}{*}{ 7E8 } & 01 & 03 & 04 & 05 & 06 & 07 & OB & OC & OD & $0 \mathrm{E}$ & $\mathrm{OF}$ & 11 & 13 & 14 & 15 & $1 \mathrm{C}$ & $1 \mathrm{~F}$ \\
\hline & 21 & $2 \mathrm{E}$ & $2 \mathrm{~F}$ & 30 & 31 & 33 & $3 \mathrm{C}$ & 41 & 42 & 43 & 44 & 45 & 47 & 49 & $4 \mathrm{~A}$ & $4 \mathrm{C}$ & \\
\hline 7E9 & 01 & 05 & OC & OD & 21 & 30 & 31 & 42 & 45 & & & & & & & & \\
\hline
\end{tabular}

The corrections of acceleration are shown in Figure 5. As revealed by the data, when the vehicle tracking system is placed flatwise inside the vehicle, an acceleration of around $0.9 \mathrm{~m} / \mathrm{sec}^{2}$ presents even if the vehicle does not accelerate. When acceleration is corrected by the Formula (1) to dispel the effect of gravity on the vehicle, the corrected acceleration maintains at around zero without exception in times of idle speed.

The actual voltage curves are shown in Figure 6. The voltage is classified into two categories. Therefore, to incorporate the advantages of both types of data, this vehicle tracking system puts forward the method for voltage correction. Test result data can be seen in the Figure, the voltages stay in the normal interval, so no abnormal voltage is detected during the test.

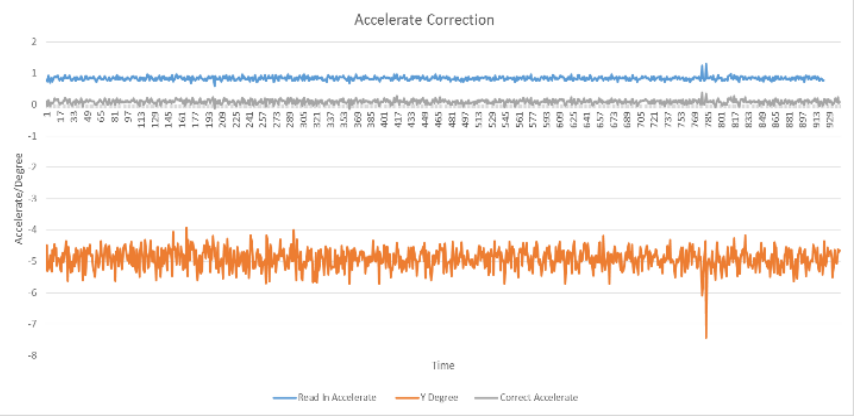

Fig. 5: A Diagram of Acceleration Differences before and after Correction.

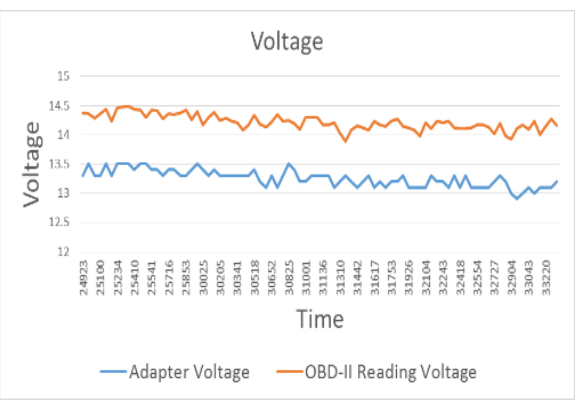

Fig. 6: A Voltage Data Graph.

Table 2 present the comparison of fuel level in the test. As can be seen the data at zero speed is relatively stable and useful for more accurate identification of the fuel state. It also verifies the feasibility and correctness for this tracking system to recognize the fuel state by capturing the fuel level at zero speed.

Voice test results are shown in Table 3. In the pure environment of target voice, the decibel meter is used to measure the sound with an average sound level of 55 DB and 65 DB. During all the ten consecutive tests, the tests mixed with the noise and target voice whose sound levels differ by $10 \mathrm{DB}$, the rate for successful identification ranges from $70 \%$ to $100 \%$.

Table 2: A Comparison of Fuel Level Data Differences by Speed-based Sampling

\begin{tabular}{|c|c|c|c|c|c|}
\hline & Max.Diff. & Mean & Variance & Std. Deviation & Coef. Variance \\
\hline All Data (Before Refuel) & 36.470 & 59.992 & 21.909 & 4.680 & 0.078 \\
\hline Speed 0 Only (Before Refuel) & 10.588 & 60.677 & 11.280 & 3.358 & 0.055 \\
\hline All Data (After Refuel) & 8.235 & 93.176 & 5.797 & 2.407 & 0.025 \\
\hline Speed 0 Only (After Refuel) & 3.137 & 94.183 & 1.477 & 1.215 & 0.012 \\
\hline
\end{tabular}

Table 3: The Successful Identification Rates during Ten Consecutive Flash Light Tests

\begin{tabular}{|c|c|c|c|c|c|}
\hline & $\begin{array}{c}\text { Without } \\
\text { Noise }\end{array}$ & $\begin{array}{c}\text { Engine Voice } \\
45 \mathrm{db}\end{array}$ & $\begin{array}{c}\text { Dialogue Voice } \\
45 \mathrm{db}\end{array}$ & $\begin{array}{c}\text { Cooler Fans Voice } \\
45 \mathrm{db}\end{array}$ & $\begin{array}{c}\text { Music Voice } \\
45 \mathrm{db}\end{array}$ \\
\hline Flash Light 55db & $100 \%$ & $90 \%$ & $70 \%$ & $100 \%$ & $90 \%$ \\
\hline Flash Light 65db & $100 \%$ & $90 \%$ & $90 \%$ & $100 \%$ & $90 \%$ \\
\hline
\end{tabular}


The states of left and right turns can be identified in two ways. First method for identification is to detect the continuous left and right turns. Second method is to compare the stable angles of the vehicle running straight. These two methods are used to test the state of making a turn. The results are shown in Table 4, indicating both methods can accurately identify left and right turns in this experimental environment.

The applications, based on the above-mentioned introduction and experimental states, are shown in Figure 7. The states of left and right turns can be combined with flash light voice. By this the administrator will know whether the driver turns on the flash light when he makes a turn or switches to another lane. Updated on abnormal messages about voltage and fuel level, the administrator can cope with the abnormal situations in advance. These states and applications mentioned above will increase the fleet's security at work and reduce extra expenditure arising from breakdown on the way.

Table 4: The Results of Left/Right Turn Identification

\begin{tabular}{|c|c|}
\hline & Success Rate \\
\hline Continue Turn Left & $100 \%$ \\
\hline Continue Turn Right & $100 \%$ \\
\hline Stable Angle Turn Left & $100 \%$ \\
\hline Stable Angle Turn Right & $100 \%$ \\
\hline
\end{tabular}

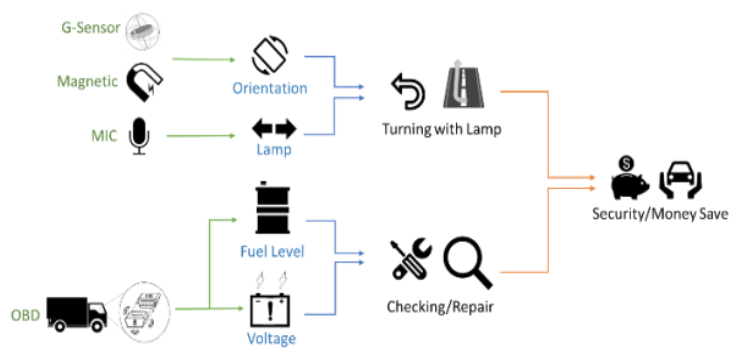

Fig. 7: States and Applications.

\section{Conclusions}

This paper presents a software vehicle tracking system which has been verified through practical operations and tests. Its functions include automatically building Bluetooth connection, reading OBD-II diagnostic messages, mobile phone sensor data, and GPS location information, identifying various states, storing data, and supporting wireless remote communication. Specific experiments have been made to ensure a high success rate of over $70 \%$ for various state identification. It is equipped with a mechanism for reconnection and another mechanism for error processing. It is sufficiently stable for long-time continuous reading performances. In the near future, apart from adjusting algorithms to improve identification precision, this vehicle tracking system will also further integrate the existing algorithms in this laboratory, expand the current functions by incorporating more diverse modules, and improve this system to a higher level.

\section{References}

[1] E/E Diagnostic Test Modes, SAE Standard J1979-2006.

[2] M. Giacobbe, A. Puliafito, and M. Villari, "A service oriented system for fleet management and traffic monitoring," 2010 IEEE Symposium on Computers and Communications (ISCC), pp. 784-786, June 2010.

[3] C.E. Lin, Y.S. Shiao, C.C. Li, S.H. Yang, S.H. Lin, and C.Y. Lin, "Real-Time Remote Onboard Diagnostics Using Embedded GPRS Surveillance Technology," IEEE Transactions on Vehicular Technology, vol. 56, no. 3, pp. 1108-1118, May 2007.

[4] G. Albertengo, W. Buttazzo, A. Tragno, M. Ricca, A. Bragagnini and R. Quasso, "Smartphone enabled connected vehicles pave the way to intelligent mobility," Proceedings of World Telecommunications Congress(WTC) 2014, pp. 1-6, June 2014.

[5] F. Beritelli, S. Casale, A. Russo, and S. Serrano, "An Automatic Emergency Signal Recognition System for the Hearing Impaired," Published in 2006 IEEE 12th Digital Signal Processing Workshop and 4th IEEE Signal Processing Education Workshop, pp. 179-182, Sept. 2006.

[6] J. Dai, J. Teng, X. Bai, Z. Shen, and D. Xuan, "Mobile phone based drunk driving detection," 2010 4th International Conference on Pervasive Computing Technologies for Healthcare, pp. 1-8, March 2010.

[7] L.J. Douglas. (September2006). Decimation-in-time (DIT) Radix-2 FFT. OpenStax CNX. Available: http://cnx.org/contents/ce67266a-1851-47e4-8bfc-82eb447212b4@7. 